\title{
Entropy Generation and Human Aging: Lifespan Entropy and Effect of Diet Composition and Caloric Restriction Diets
}

\author{
Carlos A. Silva and Kalyan Annamalai \\ Department of Mechanical Engineering, Texas A\&M University, 3123 TAMU, College Station, TX 77843-3123, USA \\ Correspondence should be addressed to Kalyan Annamalai, kannamalai@tamu.edu
}

Received 21 January 2009; Accepted 23 June 2009

Recommended by Ali Akbar Saboury

\begin{abstract}
The first and second laws of thermodynamic were applied to statistical databases on nutrition and human growth in order to estimate the entropy generation over the human lifespan. The calculations were performed for the cases of variation in the diet composition and calorie restriction diets; and results were compared to a base case in which lifespan entropy generation was found to be $11404 \mathrm{~kJ} / \mathrm{K}$ per $\mathrm{kg}$ of body mass, predicting a lifespan of 73.78 and 81.61 years for the average male and female individuals respectively. From the analysis of the results, it was found that changes of diet $\%$ of fat and carbohydrates do not have a significant impact on predicted lifespan, while the diet $\%$ of proteins has an important effect. Reduction of diet protein $\%$ to the minimum recommended in nutrition literature yields an average increase of 3.3 years on the predicted lifespan. Changes in the calorie content of the diet also have an important effect, yielding a \% increase in lifespan equal or higher than the $\%$ reduction in the diet caloric content. This correlates well experimental data on small mammal and insects, in which lifespan has been increased by diet restriction.
\end{abstract}

Copyright ( 92009 C. A. Silva and K. Annamalai. This is an open access article distributed under the Creative Commons Attribution License, which permits unrestricted use, distribution, and reproduction in any medium, provided the original work is properly cited.

\section{Introduction}

Living organisms, like humans and other mammals, constantly need to expend energy to perform physical work, to maintain body temperature in the presence of heat exchange to the environment and to produce, transport, and replace molecules that are their constituents. This energy is provided by oxidation of organic substances: Carbohydrates, fats, and amino acids, primarily introduced to the organism by feeding. Unlike conventional heat engines where the chemical energy is first converted into thermal energy and then into mechanical work, living organisms are able to convert part of nutrients' chemical energy directly into work. This is possible because the oxidation of nutrients inside the organisms, known as metabolism, proceeds through many steps allowing the capture of some of the energy in an intermediate chemical from (adenosine triphosphate or ATP) which is used almost exclusively by living things [1] for direct conversion into mechanical energy as well as for promoting many biological reactions.

In 1780, Lavoisier and Laplace measured the "heat production" of mammals by calorimetry and demonstrated that for a given nutrient both animal metabolism and combustion consumed similar quantities of oxygen $[2,3]$. Numerous experiments in humans and mammals have confirmed that thermal energy production by oxidation of carbohydrates and fat in the body is the same as the heat of combustion of these substances. Consequently, the quantity of nutrients metabolized by an organism, hence its energy input, can be estimated by measurements of breathing $\mathrm{O}_{2}$ and $\mathrm{CO}_{2}$, and the knowledge of the chemical composition of food intake.

Experiments in which the energy from food metabolism was measured and compared to the work output confirmed the efficiency of muscular work is fairly consistent at 25-30 percent [1]. This is expected because in order to convert all the available energy into work the body would have to consume the food reversibly, which implies that it would have to operate infinitely slowly; but organisms must perform work over finite periods of time. An efficiency of 25-30 percent, established over the course of evolution, seems to yield the best compromise between available energy, the biochemical engine process, and the need to ultimately perform physical work. It also implies that 75-70 percent of the food energy is disposed by outflow advection, or as heat. 
The previous concepts introduce fundamental relations between life, energy, and thermodynamics. One defining characteristic of living organisms is that they thrive on irreversibility. Thus they are not in thermal, chemical, and mechanical equilibrium with their environment. In order to sustain the process of life, organisms consume high-energy nutrients irreversibly, thus generating heat and entropy, but entropy is also transferred to the environment through various waste streams (advection) including perspiration and heat transfer via the skin, in order to maintain the biological system at fixed thermal state. If one can track the entropy generation rate per unit mass $\left(\dot{\sigma}_{m}, \mathrm{~kW} / \mathrm{kg} \mathrm{K}\right)$ of the biological system (BS) with time (aging) and estimate the integrated value

$$
\sigma_{m}(t)=\int_{0}^{t} \dot{\sigma}_{m}(t) d t
$$

then $\sigma_{m}(\mathrm{t})$ continues to increase with time as long as the organism performs its life sustaining functions involving irreversible processes.

This provokes the following: questions Is there a lifespan entropy limit? Is the process of life subject to an end similar to "the heat death of the universe" predicted by Rudolph Clausius in 1851, using lifespan energy consumption limit?

Several research efforts had presented both methodologies and results for the estimation of entropy generation during human lifespan. The main idea proposed by most of these investigations is that entropy can be related to aging through irreversible cell damage, entro pic aging or thermal denaturation, which indicates structural changes with heat thereby affecting the performance of cells. The studies also suggest that there is a limit of entropy generation that can be correlated to lifespan. The most relevant of those investigations are presented in the Literature Review section.

In general, published literature on human entropy generation relied on (2) experimental calorimetric measurements or allometric laws to account for metabolic rates and (2) energy/entropy balances in a control volume around the human body. This approach is straightforward from thermodynamics textbooks, and its results are comparable among all investigators. Our approach is rather different and is based on an availability analysis of the biochemical reactions of metabolism. In principle, life is supported by the energy provided by nutrients. If we account for the entropy generated during the metabolism of said nutrients, we potentially obtain, after some simplifications, a good approximation of the entropy generated by the process of supporting life. What is needed is an estimate of entropy generated during metabolism, including the effect of metabolic efficiency and the amount of energy required by an average human over the lifespan. The chemistry of metabolism is very well understood, and the energy requirements or food intake in humans have been extensively studied. Consequently, we have reliable information to estimate entropy generation.

The following section presents a literature review of previous investigations relevant to entropy generation in humans. Then we introduce our methodology, approximations, and calculation method. They are briefly presented here in order for this paper to be self-contained, but details were published in a previous work [4]. The results section presents the effect of diet composition and caloric restriction diets in the entropy generation in humans and compares it to the base case from our previous work. Finally, the Conclusions section summarizes our findings.

\section{Literature Review}

Hershey [5] and Hershey and Wang [6] estimated the entropy generation during human lifespan. They rationalized that for a human in resting conditions; most of the energy output from the metabolism of nutrients appears as heat, and therefore entropy generation can be approximated by the Basal Metabolic Rate (BMR) divided by the average body temperature. They also estimated the entropy exchange with the surrounding by the change of entropy in the breathing air, but it turned out to be only $2 \%$ of the "internal entropy", or entropy generated by BMR.

Hershey and Wang used BMR correlations from several sources (Shock, Robertson and Reid, and Boothby among others) and data of expected lifespan from insurance companies. They also built a whole-body calorimeter to measure heat transfer to the environment and verify BMR. Their calculations indicated that entropy produced during over lifespan was $10025 \mathrm{KJ} / \mathrm{kg} \_\mathrm{K}$ for males and $10678 \mathrm{KJ} / \mathrm{kg} \_\mathrm{K}$ for females. They defined several useful concepts like entropic age, expected lifespan, and senile death and suggested that it is not the total entropy produced during lifespan, but the rate of change on entropy production, what define the senile death. Their work is a comprehensive attempt to estimate lifespan entropy on humans. However they used BMR to estimate body entropy generation, and BMR is a resting (laboratory) metabolic rate that can differ significantly from actual (field) metabolic rates, where levels of physical activity are accounted for. Moreover, due to the difficulties associated with indirect calorimetry methods like oxygen and respiratory quotient $(\mathrm{RQ})$ the effect of the diet composition on the entropy generation was not studied.

Aoki $[7,8]$ investigated the entropy characteristics in human body under basal conditions and the effect of exercise, chills, and malarial fever on entropy generation. $\mathrm{He}$ used calorimeter experimental data from previous investigators and added the effects of heat transfer by radiation, convection, and evaporation. He also used two different body temperatures: skin temperature for heat transfer calculations and rectal temperature for internal entropy generation. Aoki found that mild and violent exercise increased entropy generation approximately 2 and 7 times, respectively, and the effect of chills and fever on entropy generation is similar to that of mild exercise. Aoki presented data for entropy production as a function of age [9] though he did not calculate entropy generation over the whole lifespan. An extrapolation of his data for basal metabolism entropy gives a value of approximately $8000 \mathrm{KJ} / \mathrm{kg} \_\mathrm{K}$.

Batato et al. [10] presented a second law analysis in which a model of the human body was established using nuclear magnetic resonance. The terms required to perform an energy balance (heat transfer, metabolic rates, etc.) were measured in test individuals. 
Batato et al. proposed that entropy generation in the human body could be divided in 3 stages: childhood (growing), healthy adult and elderly. The work was then focused on measuring the entropy on individuals in the healthy adult stage where entropy generation due to internal irreversibility was assumed to be zero and all the entropy produced was due to heat transfer, nutrient absorption, refuse rejection, and so forth. Experiments were performed over very short periods of time resulting in entropy values from $1.3 \mathrm{KJ} / \mathrm{K}$ to $11.57 \mathrm{KJ} / \mathrm{K}$ with no mass data being reported. Assuming $70 \mathrm{~kg}$ per individual and averaging the entropy values obtained $(6.92 \mathrm{KJ} / \mathrm{K})$, the entropy generation per kg of body mass obtained by Batato et al. is roughly half the value presented by Hershey and Wang $[5,6]$.

Rahman [11] calculated entropy generation in humans using the same approach as previous investigators but with a comprehensive mathematical model for the entropy fluxes. $\mathrm{He}$ accounted for free and forced convection at different air speeds, mass influx and outflux (food, water, air, waste, etc.), physical activity levels, and even for the effect of clothing. He also accounted for two body temperatures, skin and rectal, using a correlation between them to obtain a truly average body temperature for the internal entropy generation. His results were, in general, one order of magnitude higher than those of Hershey and Aoki, though they followed the same trend.

Annamalai and Puri [12] used first law analysis to obtain metabolic scaling law for biological systems and used second law to estimate entropy generation for an average human and predicted a lifespan of 77 years assuming the maximum entropy generation during as $10000 \mathrm{KJ} / \mathrm{kg} \_\mathrm{K}$.

Scaling laws (allometry) seem to describe physiological phenomena such as metabolic scaling on living organisms, but opponents of the theory argue that there are many complex factors that need to be accounted for and cannot be captured by a simple allometric equation. Supporters, however, argue that organisms evolve into configurations that maximize efficiency, reduce entropy, and naturally fall into a very narrow range of the metabolic scaling. The Constructal Design Principle proposed by Bejan [13, 14] shows how some optimal geometric forms scales to the $3 / 4$ power of their size, and explain why and how natural occurring structures (tree branches, river deltas, vascularized tissue, lightning) repeat themselves in nature.

Silva and Annamali [4] used availability analysis and the chemical reactions for metabolism to estimate entropy generation. The hypothesis is that when a CV is drawn around the human body with the boundary selected just inside the skin, so as to eliminate external irreversibilities, entropy generated can be determined by using the availability and entropy balance equations, which accounts for mass fluxes (in/out); however an alternative method would be to consider that all the activities performed by the body require energy produced by metabolism, and consequently, the entropy due to metabolic reactions should give a good approximation of entropy generated within the human body. The hypothesis presumes that nutrients not metabolized leave the system without significant change in entropy and that differences between the entropy inflow/outflow of water and air are small, as demonstrated by previous studies. Instead of the BMR, Silva et al. used the Estimated Energy Requirements (EER) from the U.S. Food and Nutrition Board (U.S. FNB) for the metabolic rates, accounting indirectly for the physical activity level (PAL), deposition of new tissue, thermal effect of food, and thermoregulation inside the individuals. Entropy generated over the lifespan of an average person was estimated to be $11404 \mathrm{KJ} / \mathrm{kg} \_\mathrm{K}$, with a rate of generation three times higher on infants than on elderly. This value is $10 \%$ to $15 \%$ higher than previous estimations from Hershey and Wang, Aoki, and Annamalai, but this was expected as the more realistic metabolic rates (EER) were used.

The approach used by Silva et al. differs from previous studies because it accounted for ATP production and considered EER (hence PALs), diet composition, and diet caloric content. A base case for the $50 \%$ percentile U.S. male and female individual was presented, as well as the PALs effect on lifespan entropy generation. In the current work, we focus on the other two aspects of the calculation, namely diet compositions and caloric restriction diets.

\section{Methodology and Approximations}

3.1. Thermodynamics. Our methodology is based on the energy and entropy balance equations as

$$
\begin{aligned}
\frac{d E_{\mathrm{cv}}}{d t} & =\dot{Q}_{\mathrm{cv}}-\dot{W}_{\mathrm{cv}}+\sum_{k}\left\{\dot{m}_{k} e_{T, k}\right\}_{i}-\sum_{k}\left\{\dot{m}_{k} e_{T, k}\right\}_{e} \\
\frac{d S_{\mathrm{cv}}}{d t} & =\frac{\dot{Q}_{\mathrm{cv}}}{T_{b}}+\sum_{k}\left\{\dot{m}_{s} s_{k}\right\}_{i}-\sum_{k}\left\{\dot{m}_{k} S_{k}\right\}_{e}+\dot{\sigma},
\end{aligned}
$$

where $Q_{\mathrm{cv}}, W_{\mathrm{cv}}, E_{\mathrm{cv}}$ and $S_{\mathrm{cv}}$ are the heat, work, total energy, and entropy in the Control Volume (CV), respectively. Also, $\dot{m}_{k}$ is the mass flux of species $k$ in and out of the CV while $e_{T, k}$ and $s_{k}$ are the specific energy (including enthalpy, kinetic and potential energy) and the entropy of species $k$.

Equation (2) use the concepts of energy conservation and entropy balance equations. When these equations are combined the availability equation is obtained:

$$
\begin{aligned}
\frac{d\left(E_{\mathrm{CV}}-T_{0} S_{\mathrm{CV}}\right)}{d t} & =\sum_{j=1}^{n} \dot{Q}_{R, j}\left(1-\frac{T_{0}}{T_{R, j}}\right)-\dot{W}_{\mathrm{CV}} \\
& +\sum_{k}\left\{\dot{m}_{k} \psi_{k}\right\}_{i}-\sum_{k}\left\{\dot{m}_{k} \psi_{k}\right\}_{e}-T_{0} \dot{\sigma}_{\mathrm{CV}}
\end{aligned}
$$

$$
\psi_{k}\left(T, P, X_{k}, T_{0}\right)=[h(T, P)+k e+p e]_{k}-T_{0} s_{k}(T, P),
$$

where $T_{0}$ is the boundary temperature of the surroundings, and $T$ is the temperature of the system of interest. The subscripts (CV) and (R) in (3) correspond to the control volume and thermal reservoirs, respectively. The flow stream availability $(\psi)$ of the inlet and exit is defined in (4).

Under normal conditions, human beings and other large organism cannot tolerate changes of body temperature 
of more than a few degrees [15] and can be considered isothermal, thus when the $\mathrm{CV}$ includes boundaries within the skin, $T_{0} \longrightarrow T_{R}$, and the first term of (3) disappears. Assuming chemical reactions inside the body to proceed at constant temperature and pressure and neglecting potential and kinetic energy, then the stream availability in equation (4) becomes

$$
\psi_{k}\left(T, P, X_{k}\right)=h_{k}(T, P)-T s_{k}\left(T, P, X_{k}\right) \approx g_{k}\left(T, P, X_{k}\right),
$$

with the Gibbs function $\mathrm{g}_{k}$, or free energy for species $k$, defined as

$$
g_{k}=h_{k}-T s_{k}
$$

For a reacting system with $k$ species, the total Gibbs function for the reactants $G_{r}$ and products $G_{p}$ is the sum of the contributions of each species. It is observed that for naturally occurring reactions, $G_{p}<G_{r}$. Thus a reduction in the free energy indicates a spontaneous reaction while an increase means that work (or energy) should be added to such reaction to proceed (e.g., many biological reactions with input of energy from ATP). For any given reaction “ $j$ ” in an ideal mixture, the change in Gibbs's free energy is given as

$$
\Delta G_{j}=G_{p, j}-G_{r, j}=\Delta G_{j}{ }^{\circ}(\mathrm{T}, \mathrm{P})+R T \ln \frac{\left[X_{p 1}\right]\left[X_{p 2}\right] \ldots}{\left[X_{r 1}\right]\left[X_{r 2}\right] \ldots} .
$$

The first term on the right is much larger than second term, so, assuming no significant changes in the concentrations $X$ of the reactants and products:

$$
\Delta G_{j}=G_{p, j}-G_{r, j} \approx \Delta G_{j}{ }^{\circ}(\mathrm{T}, \mathrm{P}),
$$

the change in free energy will allow us to find an approximate value of the entropy generation during the metabolism of nutrients.

3.2. Metabolism and Nutrients. Let us first consider the overall oxidation of glucose, a typical carbohydrate:

$$
\begin{gathered}
\mathrm{C}_{6} \mathrm{H}_{12} \mathrm{O}_{6}+6 \mathrm{O}_{2} \longrightarrow 6 \mathrm{CO}_{2}+6 \mathrm{H}_{2} \mathrm{O} \\
\Delta G_{c}=G_{p}-G_{r}=-2870 \mathrm{KJ} / \mathrm{mol},
\end{gathered}
$$

where $\Delta G_{c}$ is the change of free energy for a pure combustion process in which most of the energy is converted to random thermal energy (i.e, entropic heat) in a rather rapid reaction. Cellular respiration breaks the above process into several steps involving small changes in energy and therefore small releases of entropy, so the overall process gains a great deal in efficiency [16]. The metabolic reduction of glucose, a typical carbohydrate, through the Krebs Cycle can be summarized as

$$
\begin{gathered}
\mathrm{C}_{6} \mathrm{H}_{12} \mathrm{O}_{6}+6 \mathrm{O}_{2} \longrightarrow 6 \mathrm{CO}_{2}+6 \mathrm{H}_{2} \mathrm{O}+32 \mathrm{ATP}, \\
\Delta G_{m}=-1893 \mathrm{KJ} / \mathrm{mol},
\end{gathered}
$$

where $\Delta G_{m}$ is change of free energy for a reaction involving the production of ATP molecules. Then the work potential of ATP is

$$
W_{A T P}=\Delta G_{A T P}=\Delta G_{m}-\Delta G_{c}=977 \mathrm{KJ} / \mathrm{mol} .
$$

The chemical energy in the form of ATP is used in the body for muscular work, osmotic work, and the synthesis of new molecules, among others functions. ATP is the "energy currency" of the living organism, and it is through the free energy released by the conversion of ATP to adenosine diphosphate (ADP) that the cells can perform nonspontaneous reactions required to maintain life, for example, ion transport against concentration gradients.

Considering glucose oxidation, the metabolic efficiency is defined as

$$
\eta=\frac{\Delta G_{\mathrm{ATP}}}{\Delta G_{c}} \approx 34 \%
$$

It is apparent that only a part of the available energy has been converted into chemical energy within the ATP molecules. The difference is wasted as entropic heat.

Nutrients are modeled as carbohydrates, fats, and proteins, whose main properties are shown in Table 1. Carbohydrates in the form of glucose $\left(\mathrm{C}_{6} \mathrm{H}_{12} \mathrm{O}_{6}\right)$ constitute the major fuel of most living organisms. One molecule of glucose produces between 30 and 32 molecules of ATP as indicated in (9), resulting in a metabolic efficiency of $34.6 \%$.

Fat is the principal stored form of energy in many organisms. The amount of fat stored in a human adult of normal weight ranges from 6 to $20 \mathrm{~kg}$ and provides a large energy buffer capacity as well as the ability to survive extended periods of severe food deprivation [17]. The metabolism of one molecule of fat (palmitic acid) produces 106 molecules of ATP, preserving $32.2 \%$ or the energy available from the complete oxidation of the nutrient.

Proteins are the most abundant biological macromolecules, occurring in all cells and all parts of the cells. Proteins are composed of combinations of 20 standard components called amino acids, and they provide the blocks for synthesis of the specific molecules required by the organism.

Following biochemistry literature [17] we estimated the quatities of ATP molecules generated by the molecular respiration of each amino acid (in the acid cycle); then, using the average percentage of occurence of each amino acid in proteins, we calcualted the ATP production for an average protein. Details are given elsewhere [18]; then a weighted average of ATP molecules per amino acid was obtained. Details are given elsewhere [18]. It was found that metabolism of the average molecule of amino acid generates 8 ATP molecules, rendering an efficiency of $10.4 \%$. It is interesting to note that efficiency of protein metabolism is about $1 / 3$ of the efficiency for carbohydrates/fats and partially explains why energy is obtained from proteins in very small quantities, mostly when other sources of energy are not available. 
3.3. Statistical Databases. The energy required for bodily functions and physical activity has been summarized and tabulated by the U.S. FNB for humans from birth to adulthood. The Dietary Reference Intakes for Energy, Carbohydrate, Fiber,

Fat, Fatty Acids, Cholesterol, Protein, and Amino Acids

(Macronutrients), published in 2002 [19], contain the most updated nutrition information for US population. The values form this study, known as Dietary Reference Intake (DRI), are intended to serve as a guideline for nutrition and replace the Recommended Dietary Allowances last revised in 1989. One of the most innovative features of the DRI report is the use of the Double Labeled Water technique (DLW) for measuring the energy expenditure. DLW can be used over periods of 1 to 3 weeks on individuals leading their normal daily lives, instead of being subjected to laboratory conditions for few hours at a time [19]. Equations for energy requirements (EERs) as a function of age, weight and stature were presented in the DRI publication, as well as the recommended macronutrient distribution of food. These relations and values were used in this study to determine entropy generation.

\subsection{Approximations.}

(i) Nutrients, composed of carbohydrates, fats, and proteins will be modeled using glucose, palmitic acid, and average amino acids composition, respectively.

(ii) The metabolic efficiencies are assumed be constant over the lifespan. That is, the same rate of ATP and entropy generation per unit mole of glucose metabolized will be used for newborns and elderly.

(iii) Entropy exchanges with the environment like breathing in cold air/exhausting hot air and water evaporation through the skin are neglected. Entropy of refuse rejection is also neglected. Nutrients not metabolized (excess food) are assumed to leave the body without significant change in entropy.

(iv) EERs are used instead of basal metabolism (BMR) in order to include the entropy generated by physical activity, growing and tissue deposition, thermoregulation, and so forth.

(v) It will be assumed that energy requirements can be restricted by limiting the caloric content of the diet.

(vi) This study does not contemplate the effect of diseases, though some considerations on human health are presented.

(vii) Calculations will be made on the average male and female individuals of the U.S. population in the 50th percentile height and weight, and it will be assumed that a lowactive PAL is the best representation of the healthy average individual activity.

Once the lifetime entropy generation is determined, it will be used to predict changes in lifespan by modification on some of the initial assumptions. Two cases will be analyzed: change in the composition of the diet and change in the caloric content of the diet.

3.5. Procedure. The procedure used for numerical integration is as follows

(i) Age range from 0 to 90 years, with 0.25 year increments (trimester).

(ii) Growth data from CDC is used to obtain body size and weight as function of age for both genders and 50th percentile.

(iii) Age, stature, and weight are used to estimate EER for different PAL's, using DRI relations.

(iv) Using the recommended distribution range from U.S and the heating value of each nutrient, EER values are used to estimate food mass intake by nutrient group.

(v) The following equation, based on the previously discussed free-energy change and metabolic efficiency for every nutrient, is applied to obtain the entropy generated:

$$
\dot{\sigma}(t) \approx \sum \frac{\dot{N_{F, j}}(t)\left(1-\eta_{j}\right) \Delta G_{c, j}\left(T_{b}, P\right)}{T},
$$

where $\eta_{j}$ is the metabolic efficiency of nutrient $j$.

(vi) All data is expressed per trimester and unit total body mass.

\section{Analysis}

The specific entropy generated as a function of age is calculated using

$$
\sigma_{m}(t)=\int_{t_{\text {birth }}}^{t} \frac{\dot{\sigma}(t)}{m(t)} d t=\int_{t_{\text {birth }}}^{t} \dot{\sigma}_{m}(t) d t .
$$

4.1. Base Case. Figures 1 and 2 present the entropy generation rate and cumulative value for the base case, as calculated in our previous publication [4] and presented here for reference. The $y$-axis on Figure 2 is the entropy generation over the steps of integration, equal to one trimester or 91.5 days.

Entropy generation rate is always positive, and the rate declines rather quickly up to 18 years age; then it declines at a less steeper rate during the whole lifespan. Entropy generation rate at age 75 is $1 / 3$ of that in a 1-year-old baby. It is noted that the rate is high since specific metabolic rates are high due to large surface area to volume ratio for smaller sized infants [12]. This entropy generation trend is also present in dogs [4]. Cumulative values of entropy show a steady increase over the human lifespan. For males, the total entropy generation at 74.63 years age (average lifespan from statistics) is $11508 \mathrm{KJ} / \mathrm{kg} \_\mathrm{K}$, while females at 80.36 years age have total entropy of $11299 \mathrm{KJ} / \mathrm{kg}$ _K. These values have less than $2 \%$ difference, suggesting the idea of a fixed amount of entropy generation per unit body mass during lifetime. 


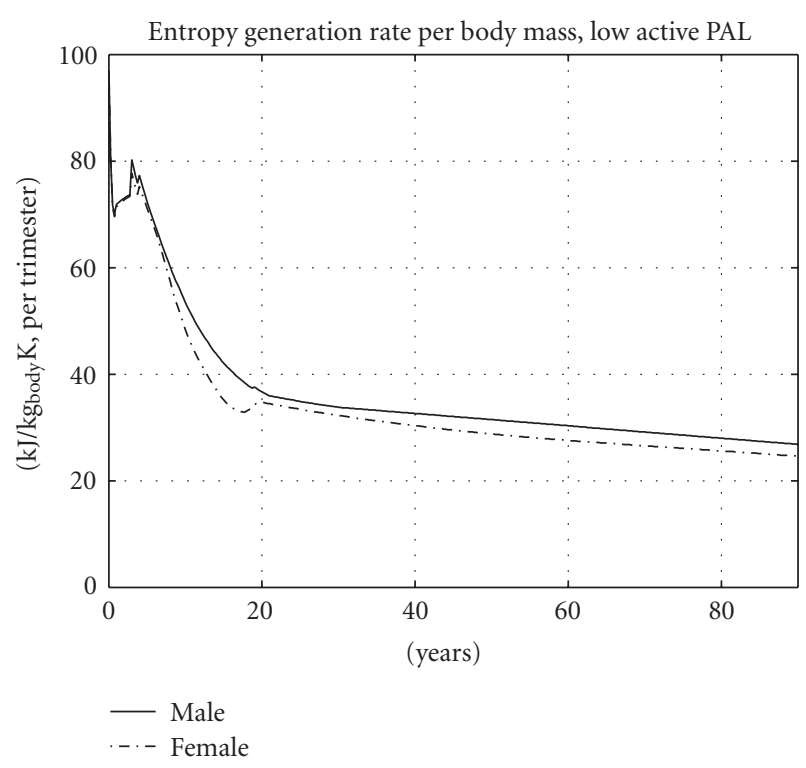

FIGURE 1: Entropy generation rate for the base case, adapted from Silva et al. [4].

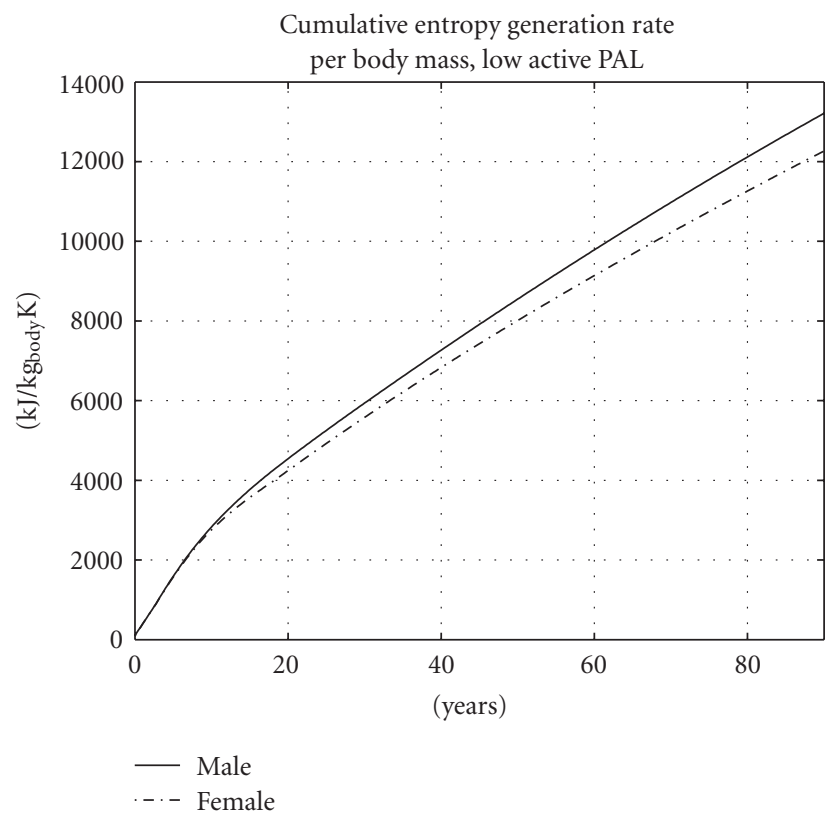

FIGURE 2: Cumulative entropy generation for the base case, adapted from Silva et al. [4].

If the average value of $11404 \mathrm{KJ} / \mathrm{kg}_{-} \mathrm{K}$ is assumed to be the limit entropy generation, lifespan for males and females is predicted to be 73.78 and 81.61 years respectively.

4.2. Case1: Change in Diet Composition. When the entropy generated by the metabolism of each nutrient is divided by its energy, an "entropy per unit energy" ratio (ENER) is obtained. For example, the heat release dur- ing metabolism of glucose is $15730 \mathrm{KJ} / \mathrm{kg}$ while entropy generated is $0.0345 \mathrm{KJ} / \mathrm{kg}_{-} \mathrm{K}$, hence the entropy to energy ratio is $0.002192 \mathrm{~K}^{-1}$. For fat and proteins the ratios are $0.002176 \mathrm{~K}^{-1}$ and $0.00323 \mathrm{~K}^{-1}$, respectively. Since metabolic efficiencies are similar for glucose and fats, the ENERs are similar. The proteins' metabolic entropy is expected to be high due to the low efficiency of the acid cycle transforming proteins to ATP. Thus thermal denaturation is severe for protein compared to glucose and fat.

The previous values suggest that for a similar profile of daily energy requirements, a change in the diet composition will change the total entropy generated. To evaluate this effect, three sets of different diet compositions were introduced to the base case, and entropy generation was recalculated.

Table 2 presents the adequate macronutrient distribution range/adequate intake from U.S. FNB. AMDR/AI are expressed in ranges that do not always add up to a $100 \%$, and the relative $\%$ of every macronutrient may change with the age. We used normalized average values over the lifespan.

The diet compositions analyzed were as follows.

(i) Base: Normal diet, all nutrients in the middle of the recommended AMDR/AI range in Table 2.

(ii) Diet 1: Carbohydrates reduced to the minimum recommended by AMDR/AI, proteins remain the same as Base case and rest of the energy supplied by fat.

(iii) Diet 2: Both carbohydrates and protein reduced to the minima AMRD/AI; with fats supplying the difference to meet EER.

(iv) Diet 3: Carbohydrates almost absent from diet, protein with an average AMDR/AI value, and the remaining energy obtained from fat.

The diet 1 can be considered as a "low-carb diet". The diet 2 corresponds to the "entropy efficient" case where protein intake is reduced to the minimum recommended based on the assumption that proteins generate the most entropy. Proteins play an important role in synthesis of new tissue (growth) so the reduction in protein \% is only introduced after 18 years age. Diet number 3 is considered an "esquimal diet", where carbohydrates are well below the minimum recommended, and most of the energy comes from fats and proteins. Table 3 shows the nutrients distribution for each set, while Table 4 presents, for each diet, the lifespan required to reach the limit entropy of $11404 \mathrm{KJ} / \mathrm{kg}$-K. The entropy generation curves for male individuals are presented in Figures 3 and 4.

Figures 3 and 4 show that changes in diet composition do not affect significantly entropy generation, and all curves look very similar. However, diet 2 where proteins were reduced to a minimum shows an interesting reduction in entropy generation with the cumulative effect of increasing the age at which limit entropy is reached from 73.78 to 77.10 years. Diet 2 is showed in the dashed line while diets 1 and 3 overlap in a single thick line. 
TABle 1: Properties of nutrients $[17,18]$.

\begin{tabular}{lccccccc}
\hline Nutrient,$j$ & Formulae & $M_{j} \mathrm{~g} / \mathrm{mol}$ & $\mathrm{HHV}_{j} \mathrm{KJ} / \mathrm{kg}$ & $h_{f, j} \mathrm{MJ} / \mathrm{kmol}$ & $\Delta \mathrm{Gc}, j \mathrm{KJ} / \mathrm{kg}$ & $S_{298, j} \mathrm{KJ} / \mathrm{kmol}$ & $\eta_{j}$, metabolic efficiency \% \\
\hline Glucose & $\mathrm{C}_{6} \mathrm{H}_{12} \mathrm{O}_{6}$ & 180 & 15630 & -1260 & -16070 & 212.0 & 34.6 \\
Amino acid & $\mathrm{C}_{4.57} \mathrm{H}_{9.03} \mathrm{~N}_{1.27} \mathrm{O}_{2.25} \mathrm{~S}_{0.046}$ & 119 & 22790 & -385 & -22430 & - & 10.4 \\
Palmitic acid & $\mathrm{C}_{16} \mathrm{H}_{32} \mathrm{O}_{2}$ & 256 & 39125 & -835 & -38465 & 452.4 & 32.2 \\
\hline
\end{tabular}

TABLE 2: Acceptable macronutrient distribution range/adequate intake (AMDR/AI) data [19].

\begin{tabular}{|c|c|c|c|c|c|c|c|}
\hline \multirow[b]{2}{*}{ Years } & & \multicolumn{2}{|c|}{ Carbohydrates } & \multicolumn{2}{|c|}{ Total fats } & \multicolumn{2}{|c|}{ Protein } \\
\hline & & $\begin{array}{c}\text { RDA/AI } \\
(\mathrm{g} / \mathrm{d})\end{array}$ & $\begin{array}{c}\text { AMDR } \\
(\%)\end{array}$ & $\begin{array}{c}\text { RDA/AI } \\
(\mathrm{g} / \mathrm{d})\end{array}$ & $\begin{array}{c}\text { AMDR } \\
(\%)\end{array}$ & $\begin{array}{c}\text { RDA/AI } \\
(\mathrm{g} / \mathrm{d})\end{array}$ & $\begin{array}{c}\text { AMDR } \\
(\%)\end{array}$ \\
\hline \multicolumn{8}{|c|}{ Infants } \\
\hline 0 & 0.5 & 60 & & 31 & & 9.1 & \\
\hline 0.5 & 1 & 95 & & 30 & & 13.5 & \\
\hline \multicolumn{8}{|c|}{ Children } \\
\hline 1 & 3 & 130 & $45-65$ & & $30-40$ & 13 & $5-20$ \\
\hline 4 & 8 & 130 & $45-65$ & & $25-35$ & 19 & $10-30$ \\
\hline \multicolumn{8}{|c|}{ Male } \\
\hline 9 & 13 & 130 & $45-65$ & & $25-35$ & 34 & $10-30$ \\
\hline 14 & 18 & 130 & $45-65$ & & $25-35$ & 52 & $10-30$ \\
\hline 19 & 30 & 130 & $45-65$ & & $20-35$ & 56 & $10-35$ \\
\hline 31 & 50 & 130 & $45-65$ & & $20-35$ & 56 & $10-35$ \\
\hline 50 & 70 & 130 & $45-65$ & & $20-35$ & 56 & $10-35$ \\
\hline$>70$ & & 130 & $45-65$ & & $20-35$ & 56 & $10-35$ \\
\hline \multicolumn{8}{|c|}{ Female } \\
\hline 9 & 13 & 130 & $45-65$ & & $25-35$ & 34 & $10-30$ \\
\hline 14 & 18 & 130 & $45-65$ & & $25-35$ & 46 & $10-30$ \\
\hline 19 & 30 & 130 & $45-65$ & & $20-35$ & 46 & $10-35$ \\
\hline 31 & 50 & 130 & $45-65$ & & $20-35$ & 46 & $10-35$ \\
\hline 50 & 70 & 130 & $45-65$ & & $20-35$ & 46 & $10-35$ \\
\hline$>70$ & & 130 & $45-65$ & & $20-35$ & 46 & $10-35$ \\
\hline \multicolumn{8}{|c|}{ Pregnancy } \\
\hline$\leq 18$ & & 175 & $45-65$ & & $20-35$ & 71 & $10-35$ \\
\hline 19 & 30 & 175 & $45-65$ & & $20-35$ & 71 & $10-35$ \\
\hline 31 & 50 & 175 & $45-65$ & & 20-35 & 71 & $10-35$ \\
\hline \multicolumn{8}{|c|}{ Lactation } \\
\hline$\leq 18$ & & 210 & $45-65$ & & $20-35$ & 71 & $10-35$ \\
\hline 19 & 30 & 210 & $45-65$ & & $20-35$ & 71 & $10-35$ \\
\hline 31 & 50 & 210 & $45-65$ & & $20-35$ & 71 & $10-35$ \\
\hline
\end{tabular}

4.3. Case 2: Change in Diet Caloric Content. Experimental data has shown that lifespan on insects and some mammals can be extended when the individuals are subjected to Caloric Restriction (CR) diets. Experiments on mice [20, 21] show that CR diets activate the Sir2 component - a conserved deacetylase - that is related to increase in lifespan. Limited experiments suggest, the same effect replicates in humans and drugs (Resveratrol) can promote it. Additional studies are available in literature [22], and the reader is recommended to consult those references if additional details are required.
For the scope of this study, two cases of CR diets will be analyzed. CR will be introduced in the base model by a $\%$ reduction in the total energy requirements over different age ranges. Table 5 shows the reduction factors used.

The reasoning behind the $\mathrm{CR} \%$ reduction is that heavy restriction should not be considered for early ages when growth (physical and physiological) is important. In teenagers $\mathrm{CR}$ is introduced to help the body adapt to the future low-energy diet regime, and the restriction is maintained below $15 \%$ up to 30 years age because this 


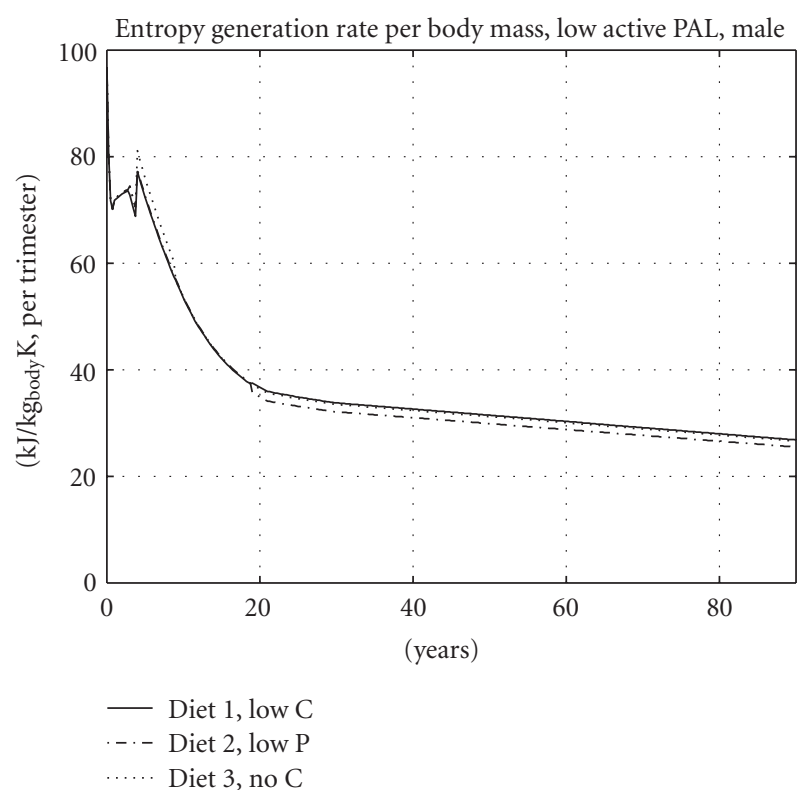

FIgURE 3: Entropy generation rate for different diet compositions.

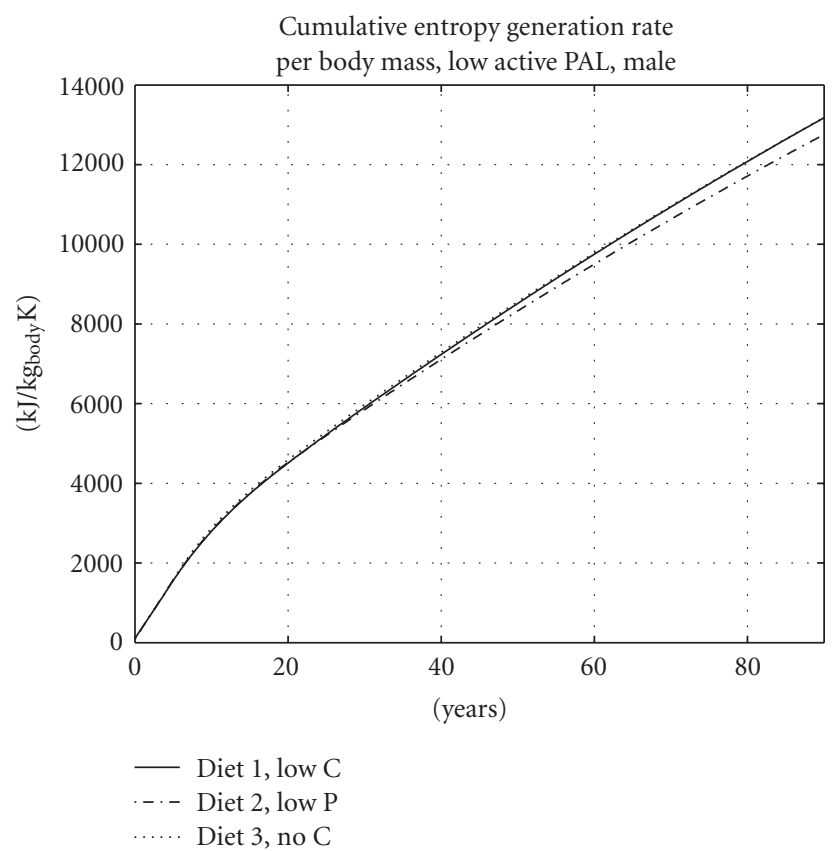

FIGURE 4: Cumulative entropy generation for different diet compositions.

age range is related to important activities like settlement and reproduction. After 30 years age, CR is enforced to keep the entropy generation as low as possible. Diet 4 represents a light restriction while diet 5 is a more heavy restriction.

Figures 5 and 6 present the plots for entropy generation and cumulative life entropy for males under the CR conditions analyzed.

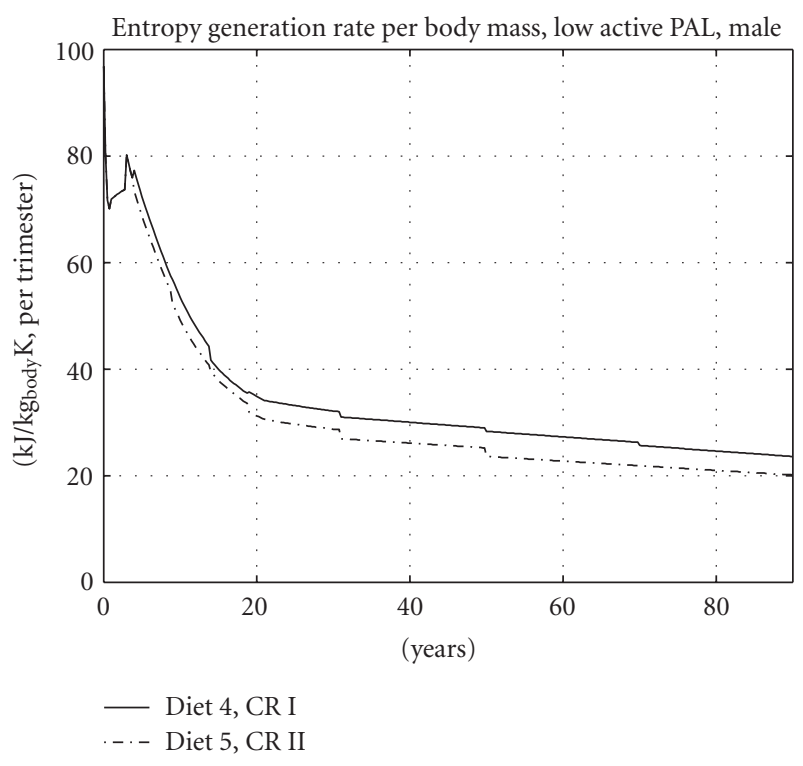

FIGURE 5: Entropy generation rate for different diet caloric content.

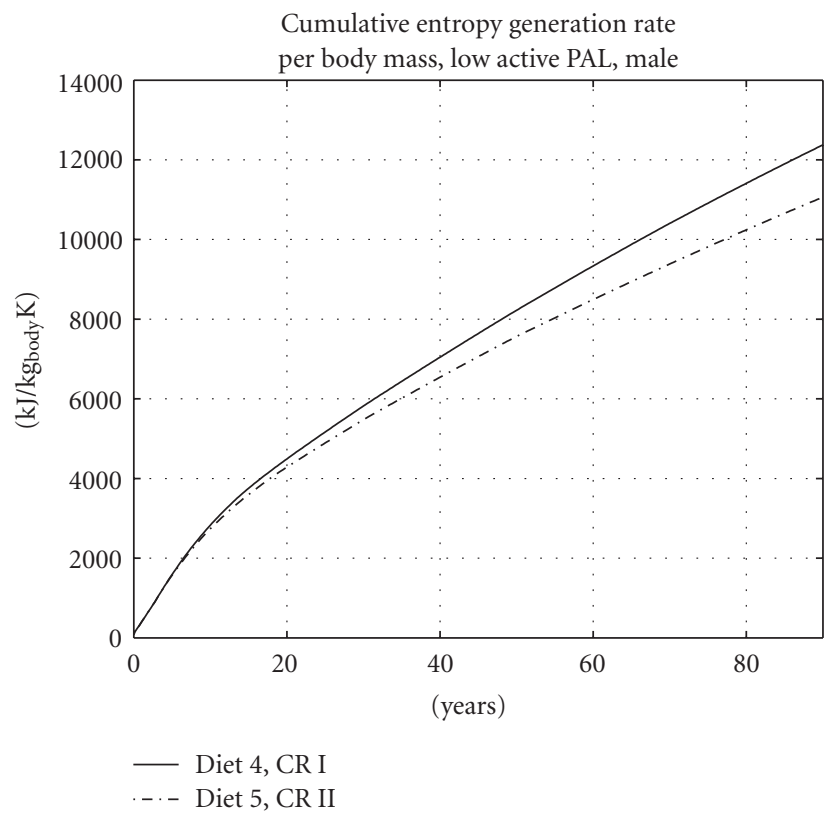

Figure 6: Cumulative entropy generation for different diet caloric content.

For humans under CR diets, the predicted lifespan to reach limit entropy is dramatically increased when compared to the base case. For the diet 4, with an average CR reduction of $8 \%$, the predicted lifespan increase is 6.16 and 7.31 years for males and females, respectively. Diet 5 has a weighted CR reduction of $18 \%$ and yields lifespan increments of 20.44 years for males and 23.96 years, females. Table 6 summarizes the results. 
TABle 3: Diet \% distribution by nutrient.

\begin{tabular}{lccc}
\hline & \multicolumn{3}{c}{ Nutrient distribution } \\
Case & Fat & Carb. & Protein \\
\hline Base & $24.5 \%$ & $52.3 \%$ & $23.2 \%$ \\
Diet 1 & $31.8 \%$ & $45.0 \%$ & $23.2 \%$ \\
Diet 2 & $43.0 \%$ & $45.0 \%$ & $12.0 \%$ \\
Diet 3 & $70.0 \%$ & $10.0 \%$ & $20 \%$ \\
\hline
\end{tabular}

TABle 4: Change in lifespan versus diet composition.

\begin{tabular}{lccc}
\hline Case & Male & $\begin{array}{c}\text { Lifespan to limit entropy }(\mathrm{y}) \\
\text { average } \\
\text { \%change }\end{array}$ & Female \\
\hline Base & 73.78 & & 81.61 \\
Diet 1 & 74.03 & $0.24 \%$ & 81.73 \\
Diet 2 & 77.10 & $4.56 \%$ & 85.35 \\
Diet 3 & 73.86 & $0.04 \%$ & 81.59 \\
\hline
\end{tabular}

TABLE 5: Calorie restriction factors ${ }^{+}$. ${ }^{+}$The stated $\%$ is reduction $\%$ compared to base case.

\begin{tabular}{lccc}
\hline & & \multicolumn{2}{c}{ Calorie restriction } \\
& Age range $(\mathrm{y})$ & Diet 4 & Diet 5 \\
\hline 0 & 8 & $0 \%$ & $0 \%$ \\
9 & 13 & $0 \%$ & $8 \%$ \\
14 & 18 & $5 \%$ & $10 \%$ \\
19 & 30 & $5 \%$ & $15 \%$ \\
31 & 50 & $8 \%$ & $20 \%$ \\
50 & 70 & $10 \%$ & $25 \%$ \\
$>70$ & & $12 \%$ & $25 \%$ \\
\hline
\end{tabular}

TABLe 6: Change in lifespan versus diet caloric content.

\begin{tabular}{lccc}
\hline Case & Male & $\begin{array}{c}\text { Lifespan to limit entropy }(\mathrm{y}) \\
\text { average \%change }\end{array}$ & Female \\
\hline Base & 73.78 & & 81.61 \\
Diet 4 & 79.94 & $8.65 \%$ & 88.92 \\
Diet 5 & 94.22 & $28.53 \%$ & 105.57 \\
\hline
\end{tabular}

\section{Conclusions}

The basic laws of thermodynamic were applied to biological systems, using a combination of theoretical background and available information from Biochemistry literature and nutrition databases. Entropy generated was determined for the metabolism of the typical components of human diet, and total entropy generation was estimated trough numerical integration for the average population.

With the defined lifespan limit entropy, $11404 \mathrm{KJ} / \mathrm{kg}_{-} \mathrm{K}$ obtained in a previous work, considered as a base case, the effects of different diet compositions and diet caloric restrictions were investigated.
The percent distribution of fats and carbohydrates in the diet does not seem to have a significant impact on the predicted lifespan to reach limit entropy. When these nutrient \% distributions are interchanged inside the recommended ranges, the average lifespan change is on the order of one trimester or less. This is expected since both fat and carbohydrates have similar entropy generation ratios. Even for an "esquimal diet" where carbohydrates are reduced to only $10 \%$ of the food intake, the lifespan entropy change is negligible.

For proteins the results are completely different. Proteins yield a very low efficiency in forming ATP by metabolism; therefore the change in the diet \% of protein causes significant entropy differences from the base case. In a diet where protein intake was reduced to the minimum recommended level, the increase in predicted lifespan was found to be 3.07 years for males and 3.62 for females.

When the caloric content of the diet is reduced, the general effect is that lifespan to reach limit entropy increases in a similar (but not the same) proportion as the timeweighted-average caloric restriction. A calorie restriction (CR) diet with a weighted average CR restriction of $8 \%$ predicted lifespan increases of 6.16 and 7.31 years for males and females respectively. These represent increments of $8.3 \%$ and $8.9 \%$ over the base case lifespan. For a diet with a CR restriction of $18 \%$, the increases were 20.44 and 23.96 years, or increments of $27.7 \%$ and, $29.36 \%$, for males, and females, respectively.

Since entropy generation rate changes with age, the period of time in which CR diet is applied seem to have a definite effect on the proportion between CR \% and lifespan increase $\%$.

To sum up, this work indicates that effective ways to increase lifespan are to reduce the diet protein intake to the minimum recommended for a healthy diet and to reduce the lifetime average caloric intake by $5-10 \%$. However, no health or disease consideration had been taken into account, and these thermodynamic calculations do not explain or prove how the process of "thermal denaturation" (heat effects on structural changes) or aging works. More research in the area is clearly needed.

\section{Acronyms}

ADP: $\quad$ Adenosine di-phosphate

AMDR/AI: Adequate macronutrient distribution range

ATP: $\quad$ Adenosine triphosphate

BMR: Basic Metabolic Rate

CDC: $\quad$ Center for Disease Control and Prevention

$\mathrm{CH}: \quad$ Carbohydrate

CV: $\quad$ Control volume

CR: $\quad$ Caloric restriction diet

DLW: Double Labelled Water

DRI: $\quad$ Daily Recommended Intake

EER: Estimated Energy Requirements

ENER: Entropy per Unit Energy

F: $\quad$ Fat

FNB: $\quad$ Food and Nutrition Board 


$\begin{array}{ll}\text { G: } & \text { Gibbs Free Energy } \\ \text { h: } & \text { Entalphy } \\ \text { HHV: } & \text { Higher heating value } \\ \text { HV: } & \text { Lower heating value of burned fuel } \\ \text { Ke: } & \text { Kinetic energy per unit mass of nutrient } \\ \text { EER: } & \text { Estimated energy requirements } \\ \text { P: } & \text { Protien } \\ \text { PAL: } & \text { Phisical Activity Levels } \\ \text { pe: } & \text { Potential energy per unit mass of nutrient } \\ \text { Q: } & \text { Heat } \\ \text { RQ: } & \text { Respiratory quotient } \\ \text { s: } & \text { Entropy } \\ \text { US FNB: U.S. Food and Nutrition Board } \\ \text { W: } & \text { Work } \\ \sigma: & \text { Entropy generation } \\ \psi: & \text { Flow stream availability. }\end{array}$

\section{References}

[1] M. Goldstein and I. F. Goldstein, The Refrigerator and the Universe, Harvard Press, 1993.

[2] F. Holmes, Lavosier and the Chemistry of Life, University of Wisconsin Press, Madison, Wiss, USA, 1985.

[3] M. Kleiber, The Fire of Life: An Introduction to Animal Energetics, Robert E. Krieger, New York, NY, USA, 1975.

[4] C. A. Silva and K. Annamalai, "Entropy generation and human aging: lifespan entropy and effect of physical activity level," Entropy, vol. 10, no. 2, pp. 100-123, 2008.

[5] D. Hershey, Lifespan and Factors Affecting It, Charles C. Thomas, Springfield, Mass, USA, 1974.

[6] D. Hershey and H. Wang, A New Age-Scale for Humans, Lexington Books, 1980.

[7] I. Aoki, "Entropy flow and entropy production in the human body in basal conditions," Journal of Theoretical Biology, vol. 141, no. 1, pp. 11-21, 1989.

[8] I. Aoki, "Effects of exercise and chills on entropy production in human body," Journal of Theoretical Biology, vol. 145, no. 3, pp. 421-428, 1990.

[9] I. Aoki, "Entropy production in human life span: a thermodynamical measure for aging," Age, vol. 17, no. 1, pp. 29-31, 1994.

[10] M. Batato, O. Deriaz, E. Jequier, and L. Borel, "Second law analysis of the human body," in Preceding of the Florence World Energy Research Symposium, Firenze, Italy, 1990.

[11] M. A. Rahman, "A novel method for estimating the entropy generation rate in a human body," Thermal Science, vol. 11, no. 1, pp. 75-92, 2007.

[12] K. Annamalai and I. Puri, Advanced Thermodynamics Engineering, CRC Press, Boca Raton, Fla, USA, 2002.

[13] A. Bejan, "The tree of convective heat streams: its thermal insulation function and the predicted 3/4-power relation between body heat loss and body size," International Journal of Heat and Mass Transfer, vol. 44, no. 4, pp. 699-704, 2001.

[14] A. Bejan, "Constructal theory: from thermodynamic and geometric optimization to predicting shape in nature," Energy Conversion and Management, vol. 39, no. 16-18, pp. 17051718, 1998.

[15] D. Haynie, Biological Thermodynamics, Cambridge Press, Cambridge, UK, 2001.

[16] K. Koehler, Biophysics, chapter 8, 1996, http://www.rwc.uc .edu/koehler/biophys/8e.html.
[17] D. Nelson and M. Cox, Lehninger Principles of Biochemistry, Worth, Worth, NY, USA, 2003.

[18] C. A. Silva, "Entropy generation in the human body. Estimation by means of energy requirements," MSME Final Report, Texas A\&M University, 2004.

[19] National Academy Press, "Dietary Reference Intakes for Energy, Carbohydrate, Fiber, Fat, Fatty Acids, Cholesterol, Protein, and Amino Acids (Macronutrients)," 2002.

[20] J. G. Wood, B. Regina, S. Lavu, et al., "Sirtuin activators mimic caloric restriction and delay ageing in metazoans," Nature, vol. 430, no. 7000, pp. 686-689, 2004.

[21] J. A. Baur, K. J. Pearson, N. L. Price, et al., "Resveratrol improves health and survival of mice on a high-calorie diet," Nature, vol. 444, no. 7117, pp. 337-342, 2006.

[22] http://en.wikipedia.org/wiki/Calorie_restriction. 

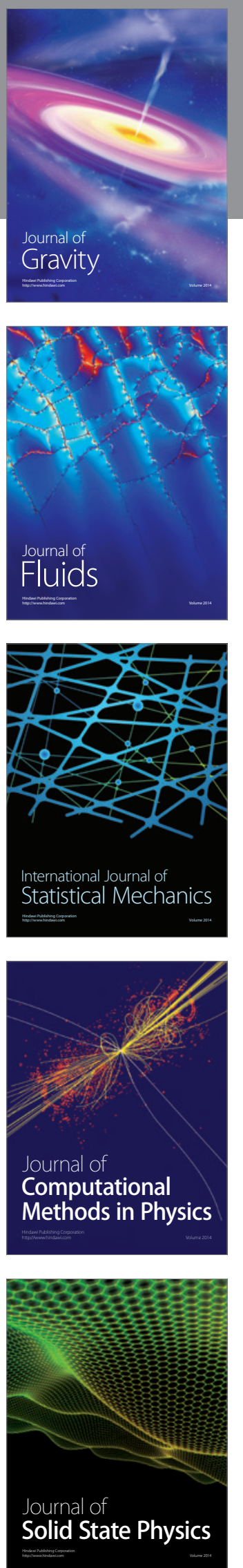

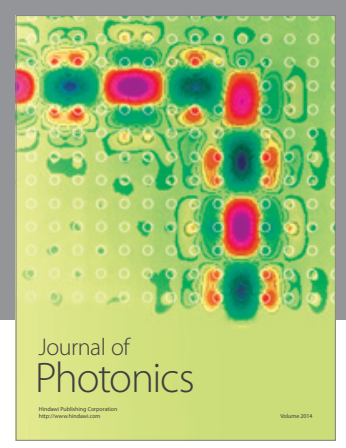

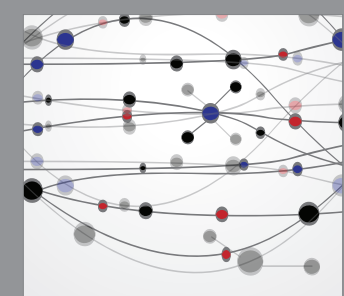

The Scientific World Journal
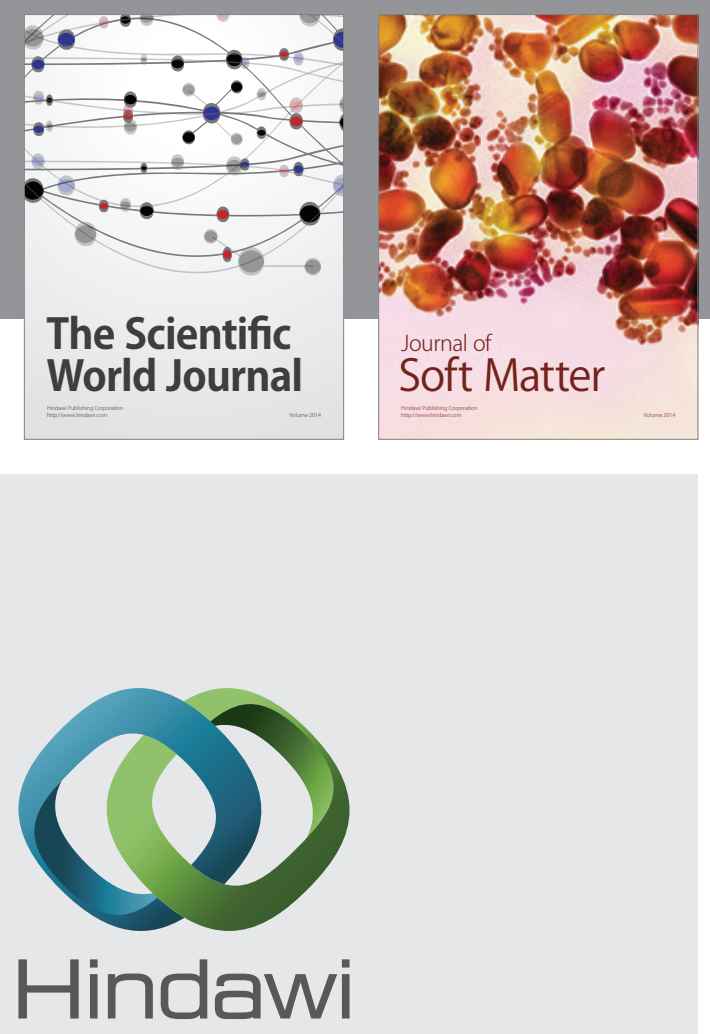

Submit your manuscripts at

http://www.hindawi.com
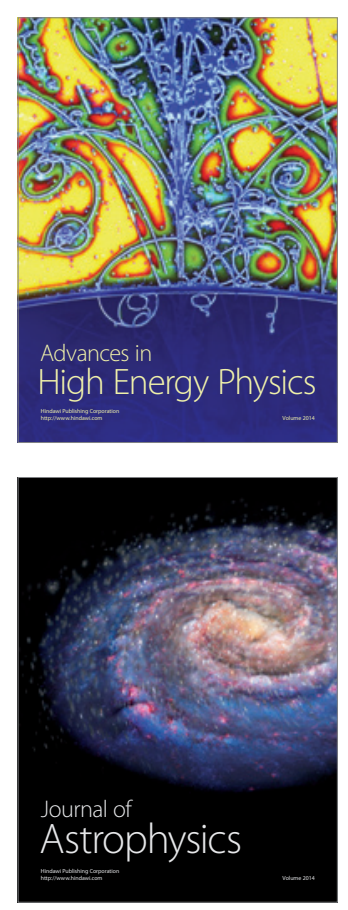
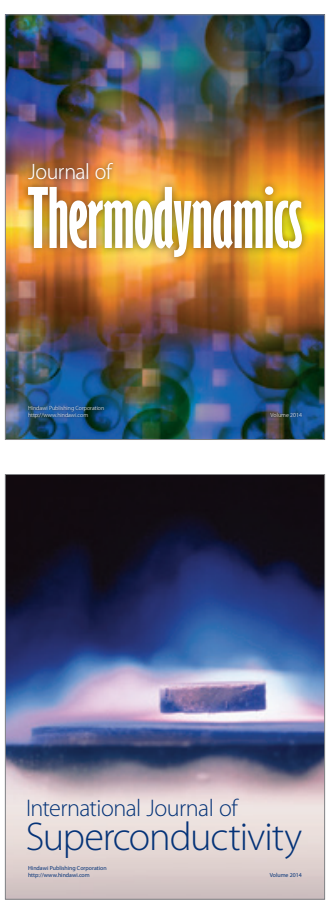
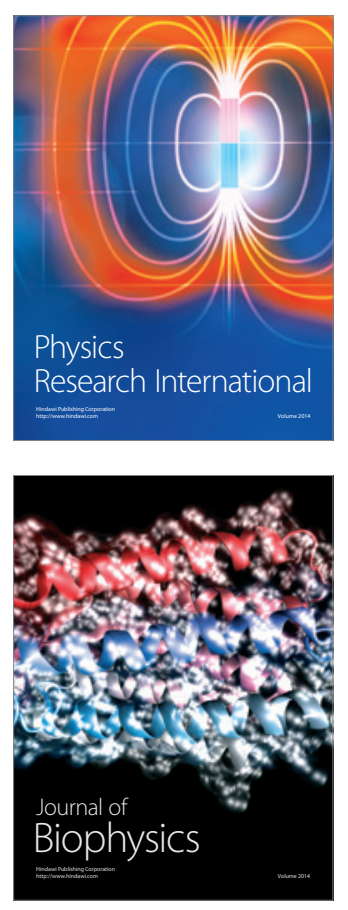
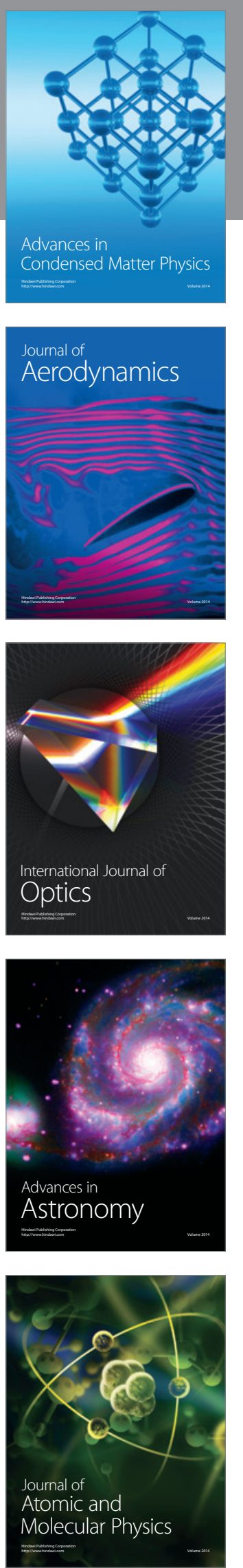